\title{
Distribución del género Bursera en el estado de Morelos, México y su relación con el clima
}

\section{Distribution of the genus Bursera in Morelos state (Mexico) and its relation to climate}

\author{
Ezequiel Hernández-Pérez ${ }^{1}$ Mario González-Espinosa² Irma Trejo $^{3}$ y Consuelo Bonfil ${ }^{1 *}$ \\ ${ }^{l}$ Departamento de Ecología y Recursos Naturales, Facultad de Ciencias, Universidad Nacional Autónoma de México. Ciudad Universitaria, Delegación \\ Coyoacán 04510 México D.F., México. \\ ${ }^{2}$ Departamento de Ecología y Sistemática Terrestres, Área de Conservación de la Biodiversidad, El Colegio de la Frontera Sur (ECOSUR), 29290 San \\ Cristóbal de Las Casas, Chiapas, México. \\ ${ }^{3}$ Instituto de Geografía, Universidad Nacional Autónoma de México, Ciudad Universitaria, Delegación Coyoacán, 04510 México D.F., México. \\ "Correspondencia: cbonfil@gmail.com
}

\begin{abstract}
Resumen. Se analizó la influencia de las variables climáticas en la distribución de 15 especies del género Bursera en Morelos, México. Se obtuvieron datos de presencia de especies y de variables climáticas de 47 estaciones meteorológicas, y se elaboraron mapas de distribución real y potencial de cada especie, por medio de un SIG; la influencia de las variables climáticas en la distribución se evaluó mediante análisis de componentes principales y de conglomerados. Las especies se distribuyen en un intervalo altitudinal de 800 a $2800 \mathrm{~m}$; la riqueza es mayor en las áreas más cálidas y relativamente más secas del sur del estado. Se identificaron 2 grandes grupos de especies: $a$ ), las de distribución restringida a sitos relativamente cálidos y b), las de distribución más amplia, incluyendo sitios menos cálidos. La precipitación, las temperaturas máxima y mínima, la evapotranspiración y su estacionalidad influyeron en la distribución de las especies. Bursera bicolor, B. bipinnata, B. cuneata, B. copallifera, B. fagaroides, B. grandifolia y $B$. glabrifolia se presentan en sitios con temperatura mínima relativamente baja $\left(5-10^{\circ} \mathrm{C}\right)$ y más húmedos, mientras que B. aptera, B. ariensis, B. lancifolia, B. linanoe, B. longipes, B. morelensis, B. schlechtendalii y B. submoniliformis se encuentran en zonas con temperaturas mínimas más altas $\left(\geq 14^{\circ} \mathrm{C}\right)$ y condiciones más secas.
\end{abstract}

Palabras clave: copal, diversidad, nicho climático, precipitación, temperatura, bosque tropical seco.

\begin{abstract}
The influence of climatic variables on the distribution of 15 species of Bursera in Morelos Mexico, was analyzed. Data on the presence of species and climatic variables from 47 meteorological stations were obtained, and maps of actual and potential distribution were made using a GIS; the influence of the main climatic variables on the distribution was analyzed by principal component and cluster analyses. The species of Bursera are found in an altitudinal range of 800-2 $800 \mathrm{~m}$; species richness is higher in the warm, relatively dry areas south of the state. Two main groups of species were distinguished: $a$ ), those having a restricted distribution, found mainly in warm sites and, $b$ ), those having a wider distribution, including relatively cool sites. Precipitation, maximum and minimum temperatures, evapotranspiration and its seasonality, all influenced the distribution of the species. Bursera bicolor, B. bipinnata, B. cuneata, B. copallifera, B. fagaroides, B. grandifolia and B. glabrifolia are found on sites having a relatively low minimum temperature $\left(5-10^{\circ} \mathrm{C}\right)$ and high precipitation, while $B$. aptera, B. ariensis, B. lancifolia, B. linanoe, B. longipes, $B$. morelensis, $B$. schlechtendalii and $B$. submoniliformis are found in areas experiencing higher minimum temperatures $\left(\geq 14^{\circ} \mathrm{C}\right)$ and relatively drier conditions.
\end{abstract}

Key words: copal, diversity, climatic niche, rainfall, temperature, tropical dry forest.

\section{Introducción}

La flora de México está considerada una de las más ricas del mundo, a lo que contribuyen su historia geológica, biogeográfica y evolutiva, su accidentada fisiografía y la enorme variedad de climas (Rzedowski,

Recibido: 08 febrero 2010; aceptado: 25 octubre 2010
1978; Villaseñor, 2003; Espejo-Serna et al., 2004). En la actualidad se estima que esta riqueza puede incluir unas 27000 especies (Sarukhán et al., 2009). El conocimiento sobre el número y la distribución geográfica de las especies vegetales en el territorio se ha acumulado rápidamente, pero el análisis de los factores que los condicionan es aún insuficiente.

Aunque el género Bursera es muy diverso y característico de la flora tropical de México, su conocimiento 
es incompleto, pues además de su sistemática complicada no se conocen bien la distribución de algunas de sus especies ni los factores que la determinan (Rzedowski et al., 2005). El grado de exploración botánica de algunas regiones del país es insuficiente, pero se puede conjeturar que la riqueza de especies de Bursera en México rebasa la cifra de 100; hasta la fecha se cuenta con registros en todos los estados de la República, con excepción de Tlaxcala. Los árboles del género Bursera son abundantes e incluso dominantes en los bosques tropicales secos de México. El género destaca tanto por el número de especies como por su abundancia, sobre todo en la vertiente del Pacífico y en la cuenca del Balsas, donde se presentan unas 80 especies (Rzedowski y Kruse, 1979, Toledo-Manzur, 1982). Su distribución está estrechamente asociada con la selva baja caducifolia (SBC; Miranda y Hernández-X., 1963) o bosque tropical caducifolio (Rzedowski, 1978), que es el bosque tropical seco de mayor extensión en México. Históricamente, se ha vinculado la distribución y diversificación del género a la expansión del bosque tropical seco en el territorio nacional (Becerra, 2005).

De acuerdo con Rzedowski et al. (2005), en Morelos se presentan 17 especies (Cuadro 1), aunque BonillaBarbosa y Villaseñor (2003) listan 23 en su catálogo de la flora de Morelos. Típicamente son árboles de tamaño bajo o medio (5-15 m); el color de su corteza varía de gris a amarillo o rojo, es delgada y con frecuencia exfoliante. La gran mayoría de las especies del género son plantas caducifolias que pierden sus hojas en la temporada seca del año y, en general, florecen al final de la misma.

La distribución de los bosques tropicales secos está estrechamente relacionada con una estacionalidad muy marcada en la precipitación, con una estación seca bien definida, de 5-7 meses de duración (Murphy y Lugo, 1995). Rzedowski (1978) ha estimado que la distribución potencial de la selva baja caducifolia abarca $14 \%$ del territorio nacional, con una extensión aproximada de $270000 \mathrm{~km}^{2}$. A finales de los años 90 se estimó que los fragmentos conservados de este tipo de vegetación se extendían alrededor de $72850 \mathrm{~km}^{2}$, y que los bosques alterados y degradados cubrían $136000 \mathrm{~km}^{2}$; es decir, la mitad del área de su distribución potencial (Trejo y Dirzo, 2000).

Los factores abióticos se encuentran entre los reguladores más importantes de la distribución de las especies (Good, 1953; Brown, 1995; Lomolino et al., 2005). Entre éstos, el clima juega un papel relevante a diferentes escalas espaciales, ya que condiciona, entre otros aspectos de índole local, los procesos de formación del suelo, las interacciones entre las especies y la frecuencia y la magnitud con que se presentan diversos fenómenos estocásticos. Por ello, las variables del clima se han usado para explicar y modelar la distribución y abundancia de las especies en la región (Guisan y Zimmermann, 2000; Arundel, 2004; González Espinosa et al., 2004). Al analizar la distribución de los biomas, también se han considerado determinantes los tipos de vegetación y la zonificación de los ecosistemas en escalas geográficas más amplias, aunque con restricciones, debido a la presencia de otros factores físicos y biológicos que operan en escalas más pequeñas (Woodward, 1987; Stephenson, 1990; Jeffree y Jeffree, 1994; Bonan, 2002; Francis y Currie, 2003; Pyke y Ehrlich, 2009).

Se han propuesto diversos modelos que permiten estimar la distribución potencial de los organismos (Guisan y Zimmermann, 2000; Elith et al., 2006); algunos cuantifican los dominios climáticos, es decir, el "espa-

Cuadro 1. Número de registros de las especies de Bursera en el estado de Morelos

\begin{tabular}{lc}
\hline Especie & No. de registros \\
\hline Bursera aptera Ramírez & 43 \\
Bursera ariensis (H.B.K.) McVaugh. et Rzed. & 12 \\
Bursera bicolor (Willd. ex Schltdl.) Engl. & 53 \\
Bursera bipinnata (DC.) Engl. & 46 \\
Bursera copallifera (DC.) Bullock. & 89 \\
Bursera cuneata (Schltdl.) Engl. & 29 \\
Bursera fagaroides (H.B.K.) Engl. & 38 \\
Bursera glabrifolia (H.B.K.) Engl. & 70 \\
Bursera grandifolia (Schltdl.) Engl. & 52 \\
Bursera lancifolia (Schltd.) Engl. & 42 \\
Bursera linanoe (La Llave) Rzed., Calderón et Medina. & 14 \\
Bursera longipes (Rose) Standl. & 23 \\
Bursera morelensis Ramírez & 22 \\
Bursera schlechtendalii Engl. & 13 \\
Bursera submoniliformis Engl. & 31 \\
\hline
\end{tabular}


cio climático" en el cual se considera que una especie sobrevive en condiciones naturales (Fischer et al., 2001). Estos modelos han mostrado su utilidad para evaluar la distribución de los organismos en análisis biogeográficos, ecológicos o de conservación (Anderson et al., 2003). Las herramientas informáticas disponibles, en especial las bases de datos y los sistemas de información geográfica (SIG), facilitan enormemente la recopilación y cartografía de la información biológica (Davis, 1994; Margules y Austin, 1994; Miller, 1994). Para modelar la variación espacial de las especies en ambientes heterogéneos se pueden utilizar funciones (por ejemplo, el kriging) que permiten interpolar el valor de una variable en sitios no muestreados en el espacio.

El objetivo de este estudio es analizar la relación entre las principales variables climáticas y la distribución de las especies más abundantes del género Bursera en el estado de Morelos para determinar su distribución potencial. Este conocimiento resultará útil en los análisis biogeográficos y en la elaboración de planes de conservación, reforestación y restauración ecológica de las $\mathrm{SBC}$, el tipo de vegetación dominante en el estado, que enfrenta fuertes presiones derivadas de la expansión de la frontera agropecuaria, los asentamientos humanos, y las posibles alteraciones derivadas del cambio climático global.

\section{Materiales y métodos}

Área de estudio. El estado de Morelos está situado en el centro sur de México, colindante con los estados de Puebla, Guerrero y el Distrito Federal (18 $20^{\circ}-19^{\circ} 08^{\prime} \mathrm{N}$ y $98^{\circ} 38^{\prime}-99^{\circ} 30^{\prime}$ O). A pesar de ocupar sólo $0.25 \%$ de la superficie de México, posee una alta diversidad, pues en su territorio se han registrado 3345 especies de plantas vasculares (Bonilla-Barbosa y Villaseñor, 2003). Predominan los climas cálidos, aunque existe un gradiente que incluye desde los climas semifríos al norte, en las faldas de las montañas más altas (con temperaturas medias entre 5 y $12^{\circ} \mathrm{C}$ ), hasta los cálidos (con temperatura media mayor a $25^{\circ} \mathrm{C}$; (García-CONABIO, 1998). La vegetación más extendida es la $\mathrm{SBC}$, en la que predominan, por su número de especies y su abundancia, las familias Leguminosae y Burseraceae (Boyás et al., 1993). En las zonas de clima templado o frío es frecuente encontrar bosques de pino, encino y zacatonales. En las partes bajas, la mayor parte de la vegetación natural ha sido reemplazada por potreros y cultivos (principalmente de maíz, frijol y calabaza de temporal, y de caña de azúcar y hortalizas en terrenos con riego; Contreras-MacBeath et al., 2006). Registros de las especies. Se elaboró una base de datos con la información de las etiquetas de los ejemplares de herbario de 15 especies del género Bursera recolectadas en Morelos (Fig. 1, Cuadro 1). La base de datos comprende los ejemplares depositados en el Herbario Nacional (MEXU), la Universidad Autónoma Metropolitana (UAM), el Centro de Educación Ambiental e Investigación de Sierra de Huautla (CEAMISH), y la Escuela Nacional de Ciencias Biológicas (ENCB). También se incluyeron registros proporcionados por la Comisión Nacional para el Conocimiento y Uso de la Biodiversidad (CONABIO), y otros obtenidos directamente durante recorridos de campo realizados en noviembre de 2004 y diciembre de 2005 en 2 áreas con escasos registros: la zona arqueológica de Xochicalco, sus alrededores y Teacalco, en el extremo oeste del estado, y en el sur, entre los poblados de Tlalquitenango y Huautla, y entre Chinameca y el Limón. De cada ejemplar se obtuvo la identidad taxonómica, nombres de los colectores, fecha y localidad de recolección, y siempre que estuvieron disponibles, la descripción del hábitat y datos geográficos (latitud, longitud, altitud). No se utilizaron ejemplares sin identificación de especie ni sin ubicación geográfica precisa. Durante los recorridos, el punto de recolección de cada ejemplar se georrefirió con un GPS (eTrexVenture, Garmin), y las coordenadas de los ejemplares de herbario se obtuvieron por interpolación en mapas topográficos (escala 1:50 000, INEGI, 1990).

Variables climáticas. La información climática se obtuvo de 47 estaciones meteorológicas del estado de Morelos

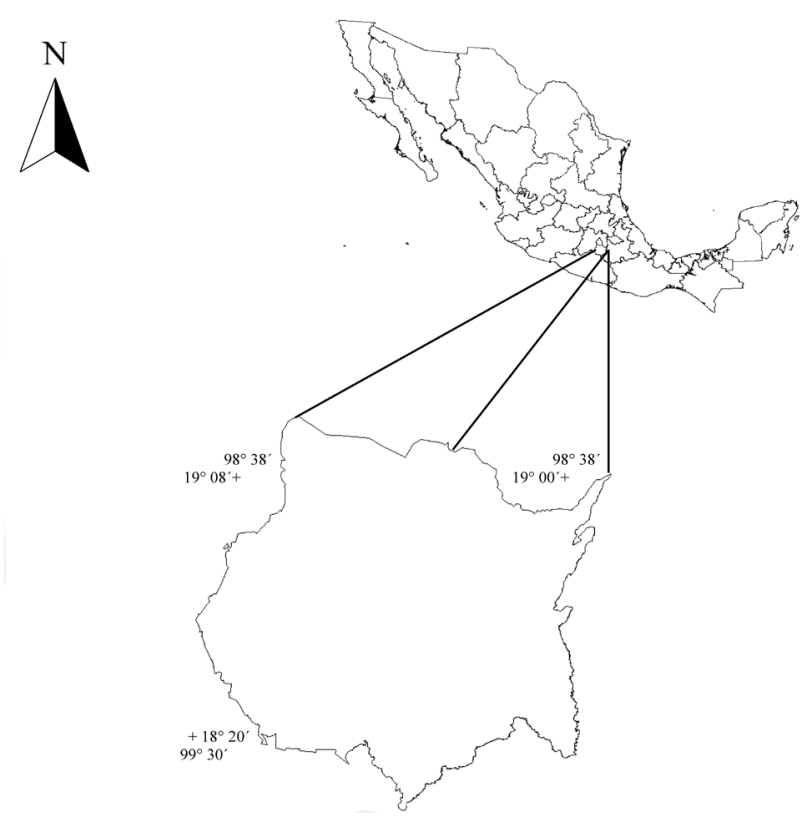

Figura 1. Ubicación del estado de Morelos en la República Mexicana. 
(Sistema de Información Climatológica, Sistema Meteorológico Nacional). Los periodos de registro fueron variables, con un máximo de 40 y un mínimo de 10 años. Se usaron los promedios de las siguientes variables: 1), temperatura máxima media anual (Tmax); 2), temperatura mínima media anual (Tmin); 3), temperatura del mes más cálido (mayo, Tmescal); 4), temperatura del mes más frío (enero, Tmesfrio); 5), precipitación en la época húmeda (Phúmeda) (meses que registran lluvia superior a $100 \mathrm{~mm}$, i.e., junio-octubre); 6), precipitación en época seca $(P s e c a)$ (precipitación de los meses que registran lluvia inferior a $100 \mathrm{~mm}, i$.e., noviembre-mayoó; 7), evapotranspiración real en la época húmeda $(E T R H)$ y en la seca (ETRS), que se estimaron con base en el modelo de evapotranspiración real anual (ETRA) de Turc (1954): ETRA $=P /\left[0.9+(P / L)^{2}\right]^{1 / 2}$, y $L=300+25 T+0.05 T^{3}$, donde $P=$ precipitación anual y $T=$ temperatura media anual; 8 ), se estimó la estacionalidad de la precipitación $(E p p)$, a partir de la diferencia entre las precipitaciones en la época húmeda y seca, y 9), se estimó la relación de proporciones de la evapotranspiración real en la época de lluvia respecto a la seca: ETRHS $=$ ETRH / ETRS (González-Espinosa et al., 2005).

Elaboración de mapas. Se elaboró la cartografía correspondiente a cada variable climática usando una matriz de datos y el método de interpolación kriging (Arc GIS 9.2, extensión Spatial Analyst), que genera mapas de contornos que describen el comportamiento de la variable en el espacio a partir de una muestra de puntos en un área geográfica. La interpolación realizada por kriging es robusta, ya que se basa en una función continua que explica el comportamiento de una variable en el espacio, y permite asociar una medida de la variabilidad de la estimación, conocida como grado de incertidumbre (Robertson, 1987; Oliver y Webster, 1990). Con los registros de presencia se elaboraron mapas de distribución real conocida de las 15 especies y su presencia se asoció con las variables climáticas antes enumeradas, para estimar la distribución potencial de cada especie. Se hicieron predicciones simples sobre la forma en que el clima afecta los patrones de distribución, usando el concepto de perfil bioclimático, i.e., el intervalo de condiciones climáticas en que se distribuyen las especies o comunidades vegetales (Box, 1981; Emanuel et al., 1985). A partir de los valores de las variables ambientales, cada perfil describe las condiciones climáticas prevalecientes en los sitios que cuentan con registros. Finalmente, se elaboró un mapa de riqueza potencial de especies de Bursera en Morelos a partir del número de especies asociadas a las variables ambientales cartografiadas.

Análisis. Se establecieron y analizaron los intervalos de las variables climáticas en que se presenta cada especie, lo que permitió distinguir grupos de especies asociadas a climas similares. Este análisis cualitativo se comparó con uno cuantitativo que se realizó mediante un análisis de conglomerados, que permitió agrupar a las especies con requerimientos climáticos similares y establecer la jerarquía de las variables para explicar la distribución (Hair et al., 1999). Adicionalmente, se realizó un análisis de componentes principales (ACP, con el programa Minitab [Release 14]), para evaluar el peso relativo de las variables ambientales consideradas en la distribución de las especies. Este análisis permite jerarquizar las variables incluidas en los componentes, al identificar aquéllas que explican el mayor porcentaje de la varianza (Wayne, 1992) y, en este caso, detectar si existen grupos de especies que tienen requerimientos climáticos similares.

\section{Resultados}

De los 570 registros georreferidos de especímenes obtenidos, alrededor del 10\% (56) corresponde a ejemplares recolectados directamente en campo, y el resto a los depositados en los siguientes herbarios: MEXU (170 registros, 29.8\%), CEAMISH (112, 19.6\%), UAM y ENCB (44 y 79 registros respectivamente, $21.6 \%$ ) y las bases de datos de la CONABIO (109, 19\%). El número de registros por especie incluyó 12 como mínimo y un máximo de 89 (Cuadro 1); las especies estudiadas fueron recolectadas a lo largo de gradientes ambientales muy amplios, entre 750 y $2340 \mathrm{~m}$ de altitud; la temperatura media anual varió entre 7.7 y $28.6^{\circ} \mathrm{C}$ y la precipitación anual entre 655 y $1885 \mathrm{~mm}$ (Cuadros 2 y 3). El análisis de distribución de la riqueza de especies muestra que las porciones norte y este del estado tienen una baja diversidad ( 1 a 3 especies), y que ésta aumenta hacia el sur y el centro (entre 8 y 10 especies). Destacan por su diversidad la sierra de Tepoztlán al N, el cañon de Lobos en la porción central, y la sierra de Huautla en el sur (Fig. 2).

En el análisis de conglomerados destaca la formación de 2 grandes grupos; el primero formado por $B$. cuneata, B. copallifera, B. bicolor, B. fagaroides, $B$. bipinnata, $B$. glabrifolia y $B$. grandifolia, y el segundo, por B. linanoe, B. longipes, B. ariensis, B. aptera, B. morelensis, B. schlechtendalii, B. submoniliformis y B. lancifolia (Fig. 3). El primero incluye especies cuya distribución abarca sitios relativamente templados (con temperaturas mínimas $\leq 11^{\circ} \mathrm{C}$, con un mínimo de entre 5 y $7^{\circ} \mathrm{C}$ ); el segundo corresponde a especies restringidas a sitios cálidos (temperatura mínima $\geq 14^{\circ} \mathrm{C}$ y máxima entre 27 y $33^{\circ} \mathrm{C}$ ).

El análisis de componentes principales (ACP) mostró que la distribución de las especies está relacionada 
Cuadro 2. Valores extremos y promedio de las variables ambientales de los sitios con registros del género Bursera en el estado de Morelos

\begin{tabular}{lccc}
\hline Variable ambiental & Mínimo & Máximo & Promedio \\
\hline Altitud & 750 & 2340 & 1828 \\
Temperatura media anual $\left({ }^{\circ} \mathrm{C}\right)$ & 7.7 & 28.6 & 18.1 \\
Temperatura máxima media anual $\left({ }^{\circ} \mathrm{C}\right)$ & 15.6 & 34 & 26.9 \\
Temperatura mínima media anual $\left({ }^{\circ} \mathrm{C}\right)$ & 7.7 & 24 & 16.7 \\
Temperatura del mes más frío $\left({ }^{\circ} \mathrm{C}\right)$ & -1 & 35.3 & 30.3 \\
Temperatura del mes más cálido $\left({ }^{\circ} \mathrm{C}\right)$ & 11.7 & 1885 & 9.1 \\
Precipitación anual $(\mathrm{mm})$ & 655 & 1743 & 1044.6 \\
Precipitación en época húmeda $(\mathrm{mm})$ & 600 & 120.9 & 1012.6 \\
Precipitación en época seca (mm) & 23.4 & 1398 & 64.4 \\
Evapotranspiración real anual $(\mathrm{mm})$ & 152.8 & 1304 & 690.8 \\
Evapotranspiración real en la época húmeda $(\mathrm{mm})$ & 594.4 & 1172 & 882.5 \\
Evapotranspiración real en la época seca $(\mathrm{mm})$ & 46.7 & 1.6 & 776.3 \\
Estacionalidad anual de la evapotranspiración real & 0.83 & 1701 & 1.3 \\
Estacionalidad de la precipitación $(\mathrm{mm})$ & 543.6 & 948.2 \\
\hline
\end{tabular}

con 6 variables del clima, contenidas en 2 componentes que explican el $70.5 \%$ de la varianza total (Cuadro 4). El primero, que explica el 50.4\%, se asocia negativamente con la precipitación -tanto en la época húmeda como en la seca y con su estacionalidad-, y positivamente con la temperatura -temperaturas máxima y mínima media anual y la temperatura media del mes más cálido-. El segundo componente (20.1\% adicional de la varianza) se asocia positivamente con la evapotranspiración real en la época seca y con la temperatura del mes más frío, y negativamente con la estacionalidad anual de la evapotranspiración.

Los resultados del ACP muestran que $B$. aptera, $B$. ariensis y $B$. morelensis se encuentran en sitios muy cálidos, secos y estacionales; las 3 se localizan en el área delimitada por valores positivos del primer componente y valores del segundo superiores a -2 (Fig. 4A). Al igual que éstas, el resto de las especies del grupo (B. lancifolia, $B$. submoniliformis, $B$. schlechtendalii, $B$. longipes y $B$. lancifolia) se encuentran restringidas a sitios con temperaturas altas, aunque toleran temperaturas ligeramente menores; B. lancifolia y en menor medida B. longipes muestran mayor amplitud climática, pues tienen registros en valores más negativos de ambos ejes de ordenación (Figs. 4B y C). En conjunto, estas 8 especies se encuentran limitadas en su distribución a sitios con temperaturas promedio (mínimas y máximas anuales) relativamente altas.

Bursera glabrifolia, B. grandifolia, B. bipinnata, $B$. fagaroides, $B$. copallifera y B. bicolor tienen menor especificidad climática y presentan un intervalo amplio de valores (i.e., se distribuyen en los 4 cuadrantes, Figs. 4E y 4F). Sin embargo, B. bicolor, B. copallifera y B. bipinnata no se encuentran en sitios relativamente frescos y húmedos y con temperaturas bajas del mes más frío, es decir valores muy negativos del primer componente. Por el contrario, B. cuneata sólo se localiza en valores negativos del primer componente y en un intervalo restringido del segundo, es decir, en sitios frescos, con baja estacionalidad en la evapotranspiración (Fig. 4D); en igual forma, B. fagaroides tiene registros en los valores más negativos del primer componente. Bursera glabrifolia y B. grandifolia también se asocian con baja estacionalidad en la evapotranspiración (Fig. 4F).

Una alta proporción de las especies se sobrepone en la porción central de ambos componentes, sobre todo en valores bajos (positivos o negativos) del componente 1, lo que permite suponer su coexistencia; la distribución de muchas de estas especies se encuentra limitada tan sólo por temperaturas promedio relativamente bajas hacia el norte del estado. Los mapas de distribución potencial (Fig. 5) muestran las áreas de distribución que cumplen con el perfil bioclimático de cada especie y la distribución real (i. e. los registros disponibles) de cada una.

\section{Discusión}

Este trabajo permitió reconocer las relaciones entre las principales variables climáticas y la presencia de las especies de Bursera en el estado de Morelos, aprovechando el mejor cúmulo de información disponible sobre las variables físicas y de las especies en la escala espacial abordada. Los resultados obtenidos indican relaciones claras entre grupos de especies y la amplitud de variación de algunas variables ambientales que son relevantes para la planeación de programas de conservación, restauración ecológica y el establecimiento de plantaciones de Bursera en el estado. Finalmente, la escala espacial utilizada 


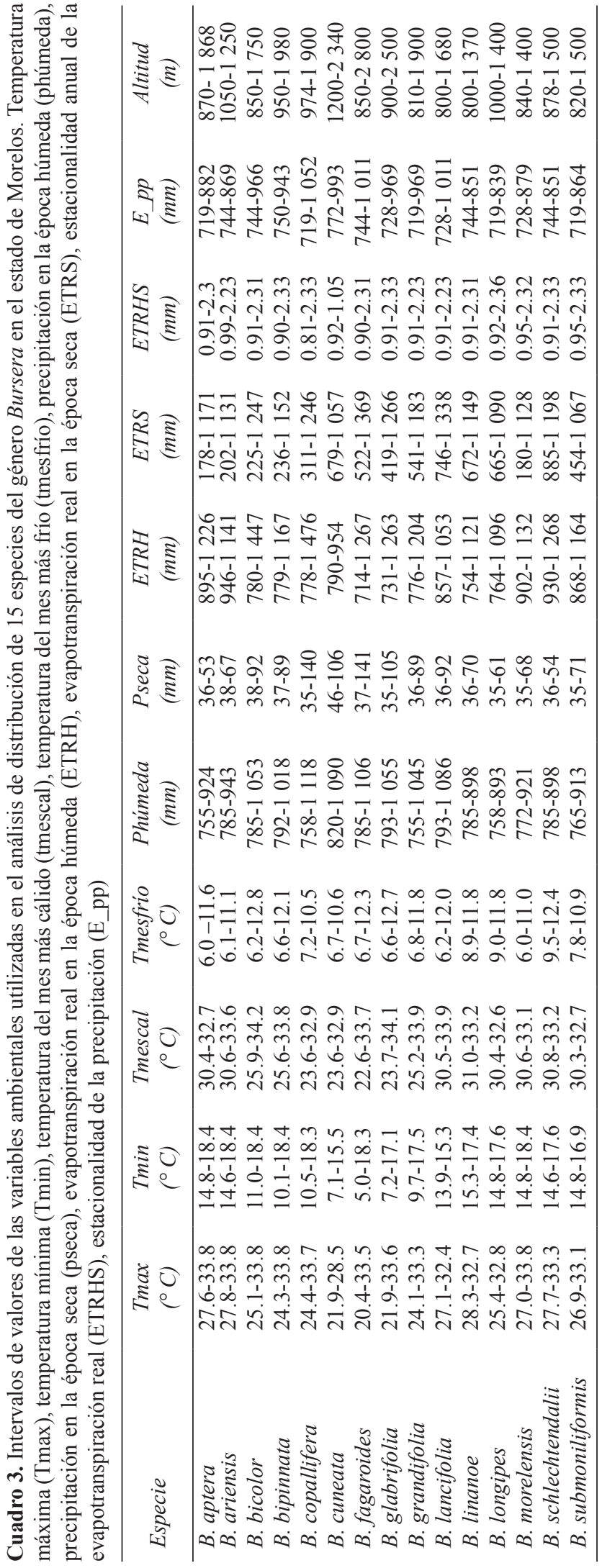

en el análisis permite vincular este estudio al análisis de escenarios de posibles afectaciones en la distribución y (o) fenología de las especies ocasionadas por el cambio climático global (Araújo y Rahbeck, 2006; Bonan, 2008).

Ninguna de las especies estudiadas es endémica del estado de Morelos y, por lo tanto, el intervalo de tolerancia a las distintas variables climáticas utilizadas podría modificarse al considerar toda su área de distribución. La recopilación y el análisis centrado en las especies de Bursera que incluyera todos los registros conocidos que definen su distribución natural es una tarea pendiente que rebasa ampliamente las posibilidades de un estudio a esta escala.

Las colecciones científicas y los inventarios biológicos constituyen herramientas fundamentales en los estudios de distribución de las especies, pero es importante considerar aspectos relevantes como el nivel de conocimiento taxonómico del grupo, los sesgos de recolecta $\mathrm{y}$, con excepciones, lo incompleto de los inventarios biológicos (Rich y Woodruff, 1992; Lawton et al., 1998; Funk y Richardson, 2002; González-Espinosa et al., 2004, Pyke y Ehrlich, 2009). Al restringir este trabajo al estado de Morelos, se logró que el conocimiento taxonómico del género, disponible en las recolecciones de herbario utilizadas, sea confiable, por los estudios previos de Toledo-Manzur (1982), Rzedowski y Guevara-Féfer (1992) y Rzedowski et al. (2004, 2005). No obstante, se ha señalado que algunas especies incluidas todavía deben ser revisadas (por ej., B. fagaroides), ya que pueden constituir complejos de especies (Rzedowski et al., 2004, 2005).

En este estudio no se dispuso de datos de inventarios cuantitativos obtenidos por muestreo de parcelas en el estado, aunque existen algunos estudios de este tipo en la cuenca del Balsas (Trejo, 1998; Pineda García et al., 2007). Una tarea importante a futuro será incluir este tipo de datos para complementar la información regional -obtenida por interpolación a partir de los registros de herbarios- con la local, contenida en parcelas de muestreo de diversos tipos de inventarios.

La mayoría de los registros disponibles para este estudio provienen del sur del estado, lo que puede deberse a la accesibilidad del área y a la concentración de los esfuerzos de investigación realizados en los últimos años, en especial a partir del establecimiento de la Reserva de la Biosfera Sierra de Huautla. Se dispone de pocos ejemplares de la porción oriental, probablemente porque la vegetación original en la región ha sido fuertemente transformada desde tiempos remotos para actividades agrícolas y pecuarias de manera continua. Existen evidencias de importantes asentamientos, al menos desde el periodo Clásico (restos en Chalcatzingo), y la agricultura que se ha practicado durante los siglos recientes ha dejado escasos fragmentos de vege- 


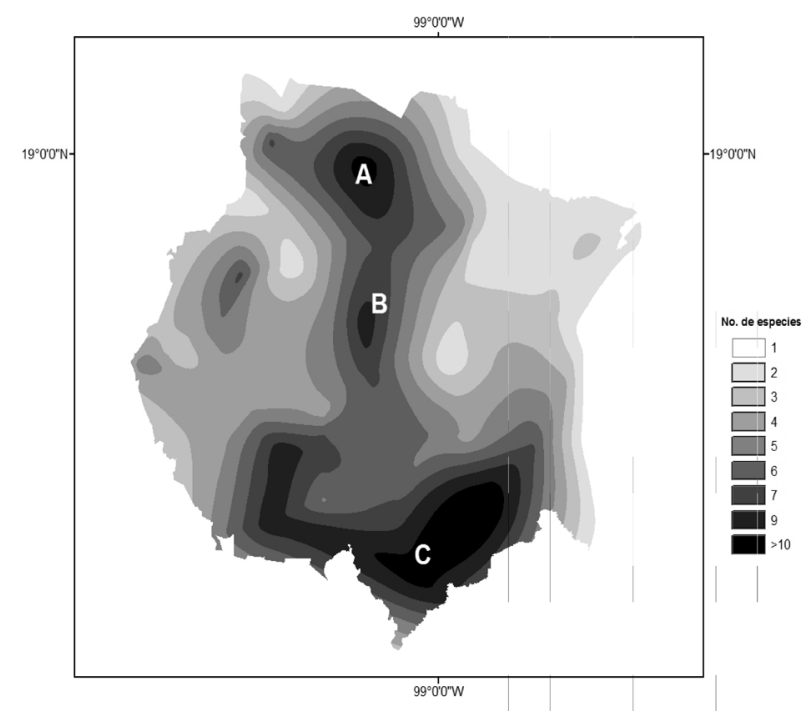

Figura 2. Riqueza de especies del género Bursera en Morelos

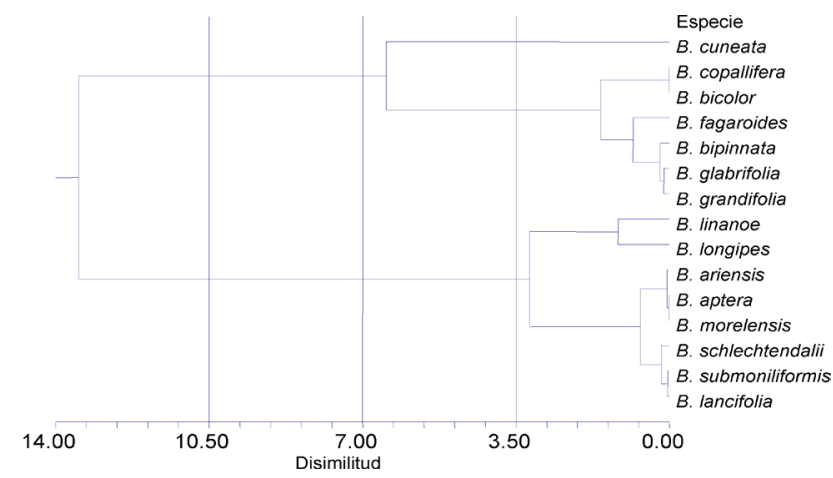

Figura 3. Agrupación de las especies de Bursera de acuerdo con su similitud climática (distancia euclidiana). tación remanente (Mazari, 1966; Oswald, 1992). Además, debe considerarse que en condiciones de relieve plano y suelos profundos y fértiles, algunas especies de selva baja son reemplazadas por otras de afinidad más xerófila (Trejo y Dirzo, 2000). La interacción entre factores que operan a escala regional, como el clima, con otros que inciden a nivel local o del paisaje, como los topográficos y edáficos, y las perturbaciones de origen antropogénico, son tema pendiente en el análisis de la distribución de las especies de Bursera.

El análisis de componentes principales (Cuadro $4 \mathrm{y}$ Figs. 4A-F) permitió establecer las variables climáticas que resultan más importantes para explicar la distribución de las especies de Bursera. En el primer eje de ordenación, la temperatura y la precipitación definen un gradiente ambiental relacionado también con la altitud. Aunque originalmente se manejó la hipótesis de la altitud como un predictor potencial de la distribución, su efecto no es directo ni uno de los más importantes.

Bursera aptera, B. ariensis y B. morelensis se distribuyen en áreas en las que prevalecen altas temperaturas, baja precipitación y acentuada estacionalidad, lo que muestra su tolerancia a condiciones de aridez. Entre ellas, B. ariensis (de la que se cuenta con relativamente pocos registros) parece tener una distribución más restringida en altitud (con un intervalo de sólo $200 \mathrm{~m}$ ) que $B$. aptera (con uno de $1000 \mathrm{~m}$, Cuadro 3). En la primera, el bajo número de registros puede ser la causa de una sobreestimación de su área de distribución potencial (Fig. 5). Bursera morelensis comparte con $B$. aptera condiciones climáticas, aunque dentro de una franja altitudinal más estrecha.

Bursera schlechtendalii, B. submoniliformis y B. lancifolia formaron un subgrupo, pero la distribución más extensa de $B$. lancifolia, -un elemento conspicuo del

Cuadro 4. Matriz de valores del análisis de componentes principales entre 11 variables climáticas y la presencia de 15 especies del género Bursera en Morelos. Se muestran en negritas las variables con mayores valores en cada componente

\begin{tabular}{lcccc}
\hline Componente & 1 & 2 & 3 & Comunalidad \\
\hline Temperatura máxima media anual $\left({ }^{\circ} \mathrm{C}\right)$ & $\mathbf{0 . 3 5 4}$ & 0.263 & 0.302 & $\mathbf{0 . 7 6 5}$ \\
Temperatura mínima media anual $\left({ }^{\circ} \mathrm{C}\right)$ & $\mathbf{0 . 3 5 0}$ & -0.092 & -0.383 & $\mathbf{0 . 6 7 5}$ \\
Temperatura del mes más cálido $\left({ }^{\circ} \mathrm{C}\right)$ & 0.346 & 0.220 & 0.199 & 0.588 \\
Temperatura del mes más frío $\left({ }^{\circ} \mathrm{C}\right)$ & 0.226 & $\mathbf{0 . 5 3 3}$ & 0.035 & 0.199 \\
Precipitación en época húmeda $(\mathrm{mm})$ & $\mathbf{- 0 . 3 6 9}$ & -0.062 & -0.442 & $\mathbf{0 . 7 5 6}$ \\
Precipitación en época seca $(\mathrm{mm})$ & $\mathbf{- 0 . 3 7 4}$ & 0.124 & -0.038 & $\mathbf{0 . 8 0 8}$ \\
Evapotranspiración real en época húmeda $(\mathrm{mm})$ & 0.300 & 0.080 & -0.430 & 0.509 \\
Evapotranspiración real en época seca $(\mathrm{mm})$ & 0.093 & $\mathbf{0 . 6 1 5}$ & 0.130 & 0.017 \\
Estacionalidad anual de la evapotranspiración & 0.223 & $\mathbf{- 0 . 4 1 7}$ & 0.222 & 0.331 \\
Estacionalidad de la precipitación & -0.348 & -0.031 & -0.520 & 0.660 \\
Altitud & -0.189 & -0.109 & -0.059 & 0.177 \\
\% de la varianza explicada & & 50.4 & 20.1 & 9.4 \\
\% acumulado & & 50.4 & 70.5 & 79.9 \\
\hline
\end{tabular}



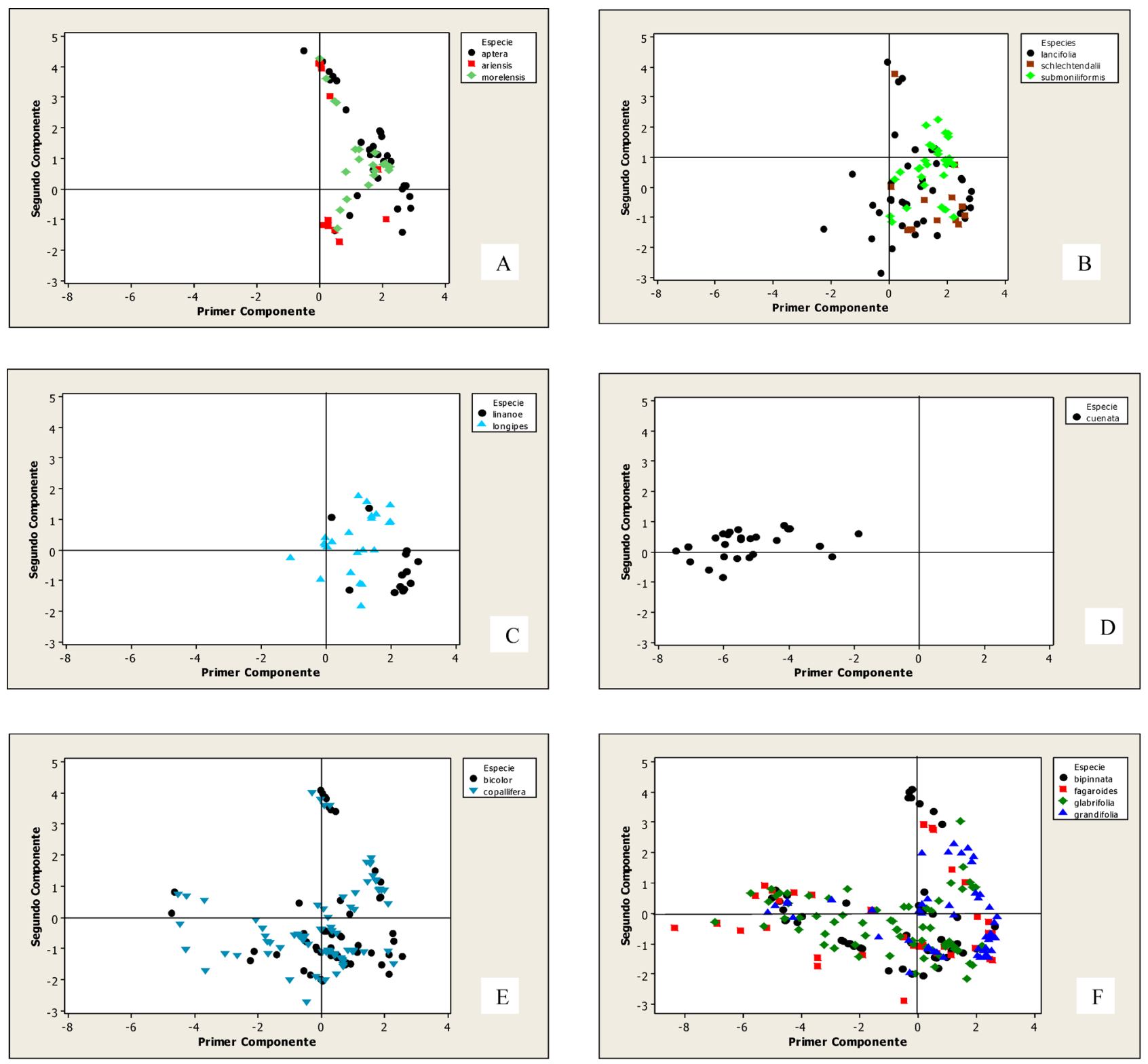

Figura 4. Distribución de las especies de Bursera en el espacio conformado por los 2 primeros componentes del ACP: A, B. aptera, B. ariensis, B. morelensis; B. B. lancifolia, B. schlechtendalii, B. submoniliformis; C, B. linanoe, B. longipes; D, B. cuneata; E, B. bicolor, B. copallifera; F B. bipinnata, B. fagaroides, B. glabrifolia y B. grandifolia.

dosel de muchas asociaciones de SBC, sobre todo en el sur del estado-, se corresponde con un intervalo altitudinal y de temperaturas amplio que incluye temperaturas más bajas que las toleradas por las otras 2 especies. Por ello, los polígonos del área de distribución de $B$. submoniliformis y $B$. schlechtendalii están incluidos dentro del de B. lancifolia (Fig. 5).

Del subgrupo formado por $B$. linanoe y $B$. longipes (Fig. 3), destaca que $B$. linanoe tiene una mayor afinidad por las temperaturas altas, y se encuentra casi exclusi- vamente en el sur del estado (excepto por un registro); ambas se distribuyen en un gradiente altitudinal relativamente estrecho (de 400 a $600 \mathrm{~m}$ ). Toledo-Manzur (1982) y Rzedowski et al. (2004, 2005) las registran en la depresión oriental del Balsas, y también se encuentran en Tehuacán-Cuicatlán. Juárez-Caballero (2008), en un análisis de la distribución de $B$. linanoe en el suroeste de Puebla, cerca del límite con Morelos y Guerrero, muestra que se distribuye principalmente en lomeríos, en zonas con temperatura media anual entre 23.8 y $25.4^{\circ} \mathrm{C}$ y preci- 


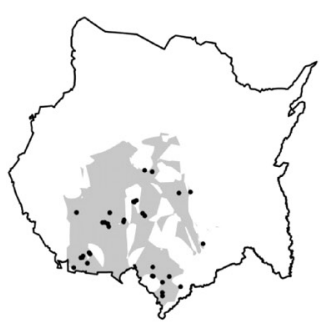

B. aptera

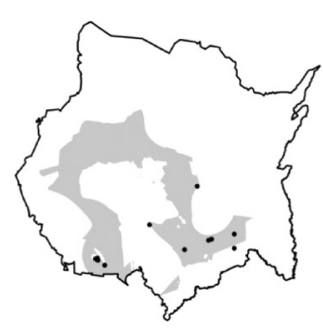

B. ariensis

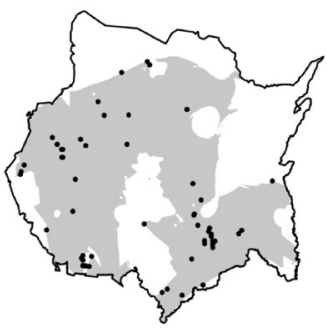

B. bicolor

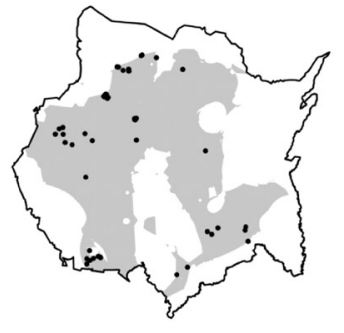

B. bipinnata

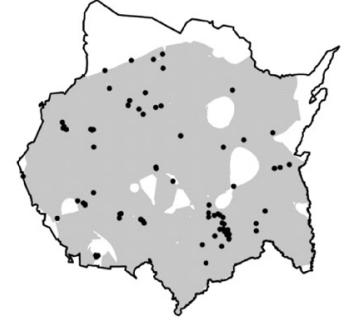

B. copallifera

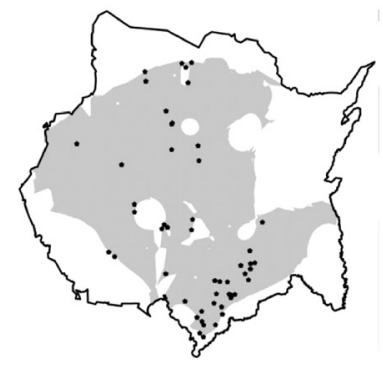

B. grandifolia

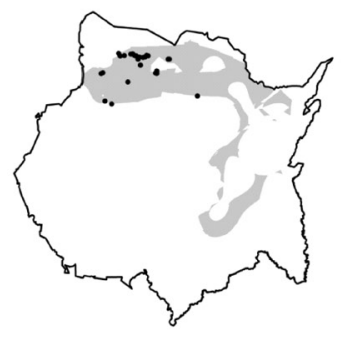

B. cuneata

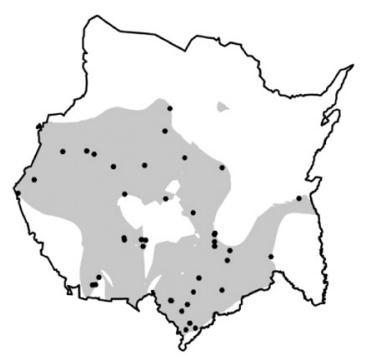

B. lancifolia

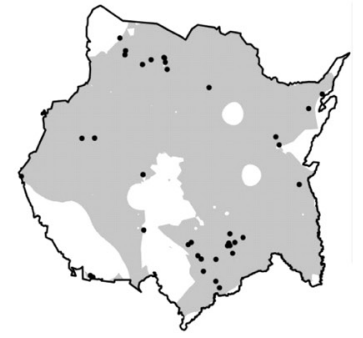

B. fagaroides

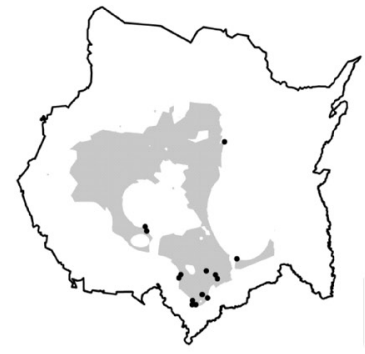

B. linanoe

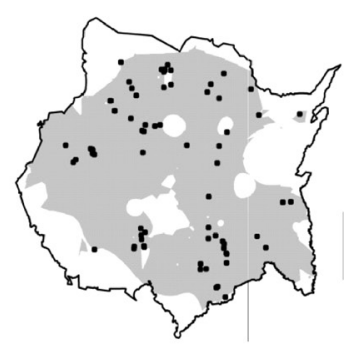

B. glabrifolia

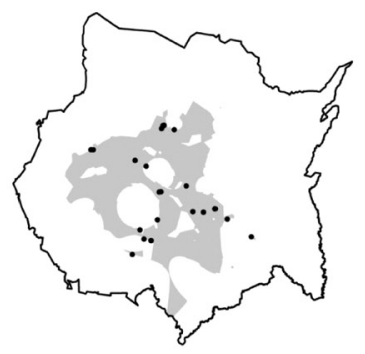

B. longipes

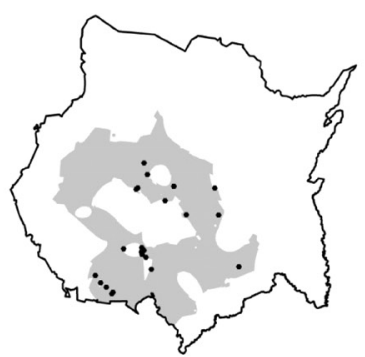

B. morelensis

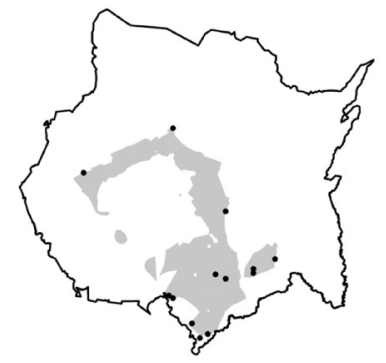

B. schlechtendalii

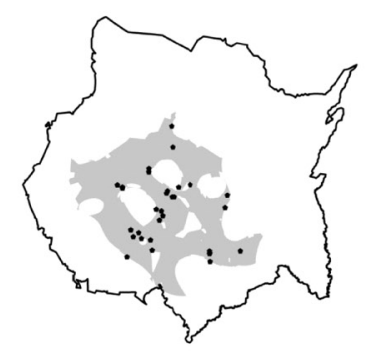

B. submoniliformis

Figura 5. Distribución potencial y ubicación de los registros de las especies de estudio en el estado de Morelos. 
pitación anual entre 736 y $802 \mathrm{~mm}$. Estas son condiciones ligeramente más cálidas y secas que las encontradas en Morelos, lo que indica que en el área de estudio esta especie ocupa ambientes relativamente mésicos dentro de su área de distribución natural. El análisis de toda la amplitud de los gradientes ambientales en que se presenta permitirá definir si el sur de Morelos corresponde a uno de los extremos de su distribución.

El otro gran grupo de especies incluye la mitad de las especies estudiadas; 4 de las 7 especies que lo conforman, B. bipinnata, B. fagaroides, B. glabrifolia y $B$. grandifolia, no presentan una gran especificidad ambiental y se distribuyen ampliamente, tanto sobre el territorio como en el diagrama del ACP (Figs. 4F y 5). Por ejemplo, en cuanto a valores de temperatura máxima, $B$. fagaroides y B. glabrifolia tienen registros desde 20 hasta casi $34{ }^{\circ} \mathrm{C}$; son también las especies de este grupo que se encuentran a mayores altitudes. De acuerdo con Rzedowski et al. (2004, 2005), ambas se presentan con frecuencia en áreas de transición entre SBC y bosque de encinos. Sin embargo, en el caso de $B$. fagaroides, esta amplitud puede deberse a que en ella se agrupan las 2 variedades que la conforman (Espinosa et al., 2006): $B$. fagaroides var. fagaroides, presente en el norte del estado y $B$. fagaroides var. purpusii en el sur. Guízar-Nolasco y Sánchez-Vélez (1991) hacen notar que B. grandifolia y B. glabrifolia - muy cercanas en el dendrograma- se presentan con frecuencia en sitios protegidos, tales como cañadas, barrancas húmedas o en la proximidad a cauces de arroyos temporales, y se han registrado como parte de la vegetación ribereña en el noroeste del estado (Camacho et al., 2006).

Bursera bicolor y B. copallifera, que tienen también una distribución amplia en el estado, no se encuentran en sitios más frescos, como las 4 especies del subgrupo anterior. Finalmente, la afinidad de B. cuneata por sitios más fríos y húmedos, con altitudes hasta de $2340 \mathrm{~m}$, concuerda con las observaciones de Rzedowski et al. (2005), quienes la registran como un elemento del sector central del Eje Neovolcánico y la consideran como una especie común en las partes más altas de SBC en otras regiones del país.

Debe destacarse también que entre las especies de distribución más amplia se encuentran aquéllas de las que se extrae el copal (B. bipinnata y B. copallifera). Por tanto, es posible que en su distribución actual incidan tanto factores ecológicos como antropogénicos (i.e., su propagación por los grupos humanos).

El análisis de la riqueza regional de Bursera en Morelos mostró su agrupamiento en 2 grandes grupos, con 3 subgrupos en cada uno, cuya distribución es posible explicar, al menos parcialmente, con base en las variables del clima. Con la excepción de $B$. cuneata, las especies de Bursera en Morelos se distribuyen en áreas relativamente cálidas, secas y estacionales en grado variable. La riqueza de especies varía, de forma que las áreas más cálidas y relativamente secas que se ubican principalmente al sur del estado (con otros puntos en Tepoztlán y el cañón de Lobos) presentan mayor número de especies que las más frías y húmedas al norte (Fig. 2). Este patrón general en la variación de la distribución espacial de la diversidad de especies de Bursera en Morelos coincide con el detectado en comunidades de SBC por otros autores (Trejo, 1998).

En algunos mapas de distribución potencial se observa una ligera sobreestimación de las áreas de distribución de las especies (por ejemplo, B. ariensis, B. cuneata y $B$. linanoe). Esto es común cuando se usan programas que estiman la distribución potencial con algoritmos de interpolación, como GARP y Bioclim. Sin embargo, el área de distribución potencial de la mayoría de las especies siguió de cerca los límites del polígono definido por las localidades con registros.

Los resultados de este estudio aportan elementos para identificar aspectos pendientes en torno a la biogeografía y sistemática (por ejemplo, las relaciones entre la afinidad climática de las especies y su agrupamiento filogenético,), la ecología (relación entre la diversidad de las especies de Bursera y otras especies arbóreas a lo largo de gradientes climáticos y edáficos) y la conservación de este grupo (identificación de áreas de alta diversidad). Así, puede destacarse que en cada uno de los 2 grandes grupos obtenidos del análisis de agrupación (Fig. 3) predominan especies de 1 de las 2 secciones en que se ha dividido al género: en el primero destacan las de la sección Bullockia ( 5 de 7 especies), mientras que el segundo grupo está constituido mayoritariamente por especies de la sección Bursera (6 de 8 especies).

Por otro lado, el estudio de los factores determinantes de la distribución y abundancia de las especies susceptibles de aprovechamiento es un antecedente indispensable para la elaboración, estructuración y monitoreo de programas de manejo sustentable. En el caso de las especies de estudio, 4 han sido identificadas por las comunidades por su valor comercial: B. linanoe (Hersch-Martínez et al., 2004) de la que se extraen aceites esenciales, $B$. glabrifolia, usada en la elaboración de alebrijes (objetos artesanales de reciente invención; Purata et al., 2004; Hernández-Apolinar et al., 2006) y B. bipinnata y $B$. copallifera, de las que se extrae el copal.

Este estudio identifica la amplitud de las variables climáticas que en mayor medida determinan la distribución y abundancia de un conjunto de especies, sin menoscabo de la incidencia de otros factores que influyen de manera local. La intensidad de aprovechamiento que las 
comunidades pueden ejercer sobre las poblaciones de las especies mencionadas de Bursera, y de cualquier otra, sin deteriorar el recurso, depende de la prevalencia de las condiciones climáticas que definen su nicho climático (Gaston 2004; Kerr et al., 2007). En una primera instancia se ha conseguido modelar el nicho climático de cada especie en una escala espacial relevante para el análisis de escenarios de cambio climático. Futuros estudios de modelación del nicho climático de las especies, junto con evidencias experimentales de intensidades de cosecha ante variaciones ambientales controladas -incremento de la temperatura, reducción de la precipitación y aumento de la estacionalidad-, permitirán predecir su abundancia y la sustentabilidad de su aprovechamiento.

\section{Agradecimientos}

E.H.P. contó con una beca de CONACYT durante el desarrollo de la investigación. Agradecemos a Josefina Hernández por su asesoría y ayuda en el manejo del SIG. P. E. Mendoza y R. Y. Evans colaboraron en el trabajo de campo. Dos revisores anónimos hicieron sugerencias valiosas que agradecemos. La investigación tuvo apoyo económico de los proyectos PAPIIT IN231808 y Manejo de Ecosistemas y Desarrollo Humano SDEI-PTID 02, de la UNAM.

\section{Literatura citada}

Anderson, R. P., D. Lew y A. T. Peterson. 2003. Evaluating predictive models of species distributions: criteria for selecting optimal models. Ecological Modeling 162:211-232.

Araújo, M. B. y C. Rahbeck. 2006. How does climate change affect biodiversity? Science 313:1396-1397.

Arundel, C. J. 2004. Using spatial models to establish climatic limits of plant species distributions. Ecological Modeling 30:1-23.

Becerra, J. X. 2005. Timing the origin and expansion of the Mexican tropical dry forest. Proceedings of the National Academy of Sciences 102:10919-10923.

Bonan, G. B. 2002. Ecological climatology. Concepts and applications. Cambridge University Press. 678 p.

Bonan, G. B. 2008. Forests and climate change: forcings, feedbacks, and the climate benefits of forests. Science 320:1444-1449.

Bonilla-Barbosa, J. R. y J. L. Villaseñor. 2003. Catálogo de la flora del estado de Morelos. Centro de Investigaciones Biológicas. Universidad Autónoma del Estado de Morelos, Cuernavaca. 129 p.

Box, E. O. 1981. Predicting physiognomic vegetation types with climate variables. Vegetatio 45:127-139.

Boyás, J. C., M. Cervantes S., J. Javelly, M. Linares A., F. Solares
A., R. Soto E., I. Naufal T. y C. Sandoval. 1993. Diagnóstico forestal del estado de Morelos. SAGAR/ INIFAP/ CIRCE, México, D.F. 245 p.

Brown, J. H. 1995. Macroecology. University of Chicago Press, Illinois. 269 p.

Camacho, R. F., I. Trejo y C. Bonfil. 2006. Estructura y composición de la vegetación ribereña de la barranca del río Tembembe, Morelos, México. Boletín de la Sociedad Botánica de México 78:17:31.

Contreras-MacBeath, T. E., F. Jaramillo y J. C. Boyás. 2006. La diversidad biológica en Morelos: estudio del estado. Comisión Nacional para el Conocimiento y Uso de la Biodiversidad, México, D. F. 155 p.

Davis, F. W. 1994. Mapping and motoring terrestrial biodiversity using geographic information systems. In Biodiversity and terrestrial ecosystems, C. H. Chou (ed.). Institute of Botany, Academia Sinica Monograph Series 14, Taipei. p. 461-471.

Elith, J., C. H. Graham, R. P. Anderson, M. Dudík, S. Ferrier, A. Guisan, R. J. Hijmans, F. Huettmann, J. R. Leathwick, A. Lehmann, J. Li, L. G. Lohmann, B. A. Loiselle, G. Manion, C. Moritz, M. Nakamura, Y. Nakazawa, J. M. Overton, A. T. Peterson, S. J. Phillips, K. Richardson, R. Scachetti-Pereira, R. E. Schapire, J. Soberón, S. Williams, M. S. Wisz y N. E. Zimmermann. 2006. Novel methods improve prediction of species distributions from occurrence data. Ecography 29:129-151.

Emanuel, W. R., H. H. Shugart, y M. P. Stevenson. 1985. Climatic change and the broad-scale distribution of terrestrial ecosystem complexes. Climatic Change 7:29-43.

Espejo-Serna, A., A. R. López-Ferrari e I. Salgado-Ugarte. 2004. A current estimate of angiosperm Diversity in Mexico. Taxon 53:127-130.

Espinoza, D., J. Llorente y J. J. Morrone. 2006. Historical biogeographical patterns of the species of Bursera (Burseraceae) and their taxonomic implications. Journal of Biogeography 33:1945-1958.

Fisher, J., D. B. Lindenmayer, H. A. Nix, J. L. Stein y J. A. Stein. 2001. Climate and animal distribution: a climatic analysis of the Australian marsupial Trichosurus caninus. Journal of Biogeography 28:293-304.

Francis, A. P. y D. J. Currie. 2003. A globally consistent richnessclimate relationship for angiosperms. The American Naturalist 161:523-536.

Funk, V. A. y K. S. Richardson. 2002. Systematic data and biodiversity studies: use it or lose it. Systematic Biology 51:303-316.

García, E. 1998. Climas. Escala 1:1,000,000. México, Comisión Nacional para el Conocimiento y Uso de la Biodiversidad (CONABIO), México, D.F.

Gaston, K. J. 2004. Macroecology and people. Basic and Applied Ecology 5:303-307.

González-Espinosa, M., J. M. Rey-Benayas, N. Ramírez- 
Marcial, M. A. Huston y D. Golicher. 2004. Tree diversity in the northern Neotropics: regional patterns in highly diverse Chiapas, México. Ecography 27:741-756.

González-Espinosa, M., N. Ramírez-Marcial, G. Méndez-Dewar, L. Galindo-Jaimes y D. Golicher. 2005. Riqueza de especies de árboles en Chiapas: variación espacial y dimensiones ambientales asociadas al nivel regional. In Diversidad biológica en Chiapas, M. González-Espinosa, N. RamírezMarcial y L. Ruiz-Montoya (eds.). Plaza y Valdés, México, D. F. p. 81-117.

Good, R. 1953. The geography of the flowering plants, 2a. edición. Longman, Londres. p. 452.

Guisan, A. y N. E. Zimmermann. 2000. Predictive habitat distribution models in ecology. Ecological Modelling 135:147-186.

Guízar-Nolasco, E. y A Sánchez-Vélez. 1991. Guía para el reconocimiento de los principales árboles del Alto Balsas. Universidad Autónoma Chapingo, Chapingo. 207 p.

Hair, J. F., R. E. Anderson, R. E. Tatham y W. C. Black. 1999. Análisis Multivariante. Prentice Hall España, Madrid. 832 p.

Hernández-Apolinar, M., T. Valverde y S. E. Purata. 2006. Demography of Bursera glabrifolia, a tropical tree used for folk woodcrafting in southern Mexico: an evaluation of its management plan. Forest Ecology and Management 223:139-151.

Hersch-Martínez, P., R. Glass y A. A. Fierro. 2004. E1 linaloe (Bursera aloexylon (Schiede) Engl.): una madera aromática entre la tradición y la presión económica. In Productos forestales, medios de subsistencia y conservación: estudios de caso sobre sistemas de manejo de productos forestales no maderables, vol. 3, América Latina, M. N. Alexiades y P. Shanley (eds.). Centro para la Investigación Forestal Internacional (CIFOR), Bogor [Indonesia]. p. 439-462.

INEGI (Instituto Nacional de Estadística, Geografía e Informática). 1990. Cartas topográficas, escala 1:50000. Claves E14A48, E14A49, E14A58 y E14A59. Aguascalientes, Aguascalientes.

Jeffree, E. P. y C. E. Jeffree. 1994. Temperature and the biogeographical distributions of species. Functional Ecology $8: 640-650$

Juárez-Caballero, L. F. 2008. Análisis de la distribución de Bursera linanoe (Llave) Rzed, Calderón \& Medina (Burseraceae) en el sureste del estado de Puebla como base para su manejo. Tesis, Maestría. Facultad de Estudios Superiores Zaragoza, Universidad Nacional Autónoma de México, México, D. F. $78 \mathrm{p}$.

Kerr, J. T., H. M. Kharouba y D. J. Currie. 2007. The macroecological contribution to global change solutions. Science 316:1581-1584.

Lawton, J. H., D. E. Bignell, B. Bolton, G. F. Bloemers, P. Eggleton, P. M. Hammond, M. Hodda, R. D. Holt, T. B. Larsen, N. A. Mawdlsley, N. E. Stork, D. S. Srivastava y
A. D. Watt. 1998. Biodiversity inventories, indicator taxa and effects of habitat modification in tropical forest. Nature 291:72-76.

Lomolino, M. V., B. R. Riddle y J. H. Brown. 2005. Biogeography, 3a. edición. Sinauer. Sunderland, Massachusetts. 868 p.

Margules, C. R. y M. P. Austin. 1994. Biological models for monitoring species decline: the construction and use of data bases. Philosophical Transactions of the Royal Society of London 344:69-75.

Mazari, M. 1966. Bosquejo histórico del estado de Morelos. Secretaría de Educación Pública, México, D. F. 229 p.

Miranda, F. y E. Hernández-X. 1963. Los tipos de vegetación de México y su clasificación. Boletín de la Sociedad Botánica de México 23:29-47.

Miller, R. I. 1994. Mapping the diversity of nature. Chapman and Hall, London. 218 p.

Murphy, D. E. y A. E. Lugo. 1995. Dry forest of Central America and the Caribbean. In Seasonally dry tropical forest, S. H. Bullock, H. A. Money y E. Medina (eds.). Cambridge University Press. p. 9-34.

Oliver, M.A.y R. Webster. 1990. Kriging: a method of interpolation for geographical information systems. International Journal of Geographical Information Systems 4:313-332.

Oswald, U. 1992. Mitos y realidades del Morelos actual. Centro Regional de Investigaciones Multidisciplinarias, Universidad Nacional Autónoma de México, Cuernavaca, Morelos. 143 p.

Pineda-García F., L. Arredondo-Amezcua y G. Ibarra-Manríquez. 2007. Riqueza y diversidad de especies leñosas del bosque tropical caducifolio El Tarimo, Cuenca del Balsas, Guerrero. Revista Mexicana de Biodiversidad 78:129-139.

Purata, S., M. Chibnik, B. Brosi y A. López. 2004. Figuras de madera de Bursera glabrifolia H.B.K. (Engl.) en Oaxaca, México. In Productos forestales, medios de subsistencia y conservación. Estudios de caso sobre sistemas de manejo de productos forestales no maderables, M. N. Alexiades y P. Shanley (eds.). Centro para la Investigación Forestal Internacional (CIFOR), Bogor [Indonesia]. p. 415-437.

Pyke, G. H. y P. R. Ehrlich. 2009. Biological collections and ecological/environmental research: a review, some observations and a look to the future. Biological Reviews 85:247-266.

Rich, T. C. G. y E. E. Woodruff. 1992. Recording bias in botanical surveys. Watsonia 19:73-95.

Robertson, G. P. 1987. Geostatistics in ecology: interpolating with known variance. Ecology 68:744-748.

Rzedowski, J. 1978. Vegetación de México. Limusa, México, D. F. 432 p.

Rzedowski, J. y H. Kruse. 1979. Algunas tendencias evolutivas en Bursera (Burseraceae). Taxon 28:103-116.

Rzedowski, J. y F. Guevara-Féfer. 1992. Burseraceae. Flora del Bajío y de Regiones Adyacentes 3:1-46.

Rzedowski, J., R. Medina Lemos y G. Calderón de Rzedowski. 
2004. Las especies de Bursera (Burseraceae) en la cuenca superior del río Papaloapan (México). Acta Botanica Mexicana 66:23-151.

Rzedowski, J., R. Medina Lemos y G. Calderón de Rzedowski. 2005. Inventario del conocimiento taxonómico de la diversidad y del endemismo regionales de las especies mexicanas de Bursera (Burseraceae). Acta Botanica Mexicana 70:85-111.

Sarukhán, J. et al. 2009. Capital natural de México. Síntesis: conocimiento actual, evaluación y perspectivas de la sustentabilidad. Comisión Nacional para el Conocimiento y Uso de la Biodiversidad, México, D. F. 100 p.

Stephenson, N. L. 1990. Climatic control of vegetation distribution: the role of the water balance. The American Naturalist 135:649-670.

Toledo-Manzur, C. A. 1982. El género Bursera (Burseraceae) en el estado de Guerrero. Tesis, Facultad de Ciencias, Universidad
Nacional Autónoma de México, México D. F. 182 p.

Trejo, I. 1998. Distribución y diversidad de las selvas bajas de México: relaciones con el clima y suelo. Tesis, Doctorado Facultad de Ciencias, Universidad Nacional Autónoma de México, México, D. F. 210 p.

Trejo, I. y R. Dirzo. 2000. Deforestation of seasonally dry tropical forest: a national and local analysis in Mexico. Biological Conservation 94:133-142.

Turc, L. 1954. Le bilan d'eau des sols: relations entre les précipitation, l'évaporation et l'écoulement. Annales Agronomiques 5:491-596.

Villaseñor, J. L. 2003. Diversidad y distribución de las Magnoliophyta de México. Interciencia 28:160-167.

Wayne, W. D. 1992. Bioestadística. Base para el análisis de las ciencias de la salud. Limusa, México, D. F. 444 p.

Woodward, F. I. 1987. Climate and plant distribution. Cambridge University Press. 188 p. 